\title{
Nucleon 3D Imaging Program with SoLID at Jefferson Lab
}

\author{
Zhiwen Zhao*t \\ Department of Physics, Duke University, Durham, North Carolina 27708, USA \\ E-mail: zwzhaodjlab.org

\section{Tianbo Liu} \\ Department of Physics, Duke University, Durham, North Carolina 27708, USA \\ Thomas Jefferson National Accelerator Facility, Newport News, VA 23606, USA \\ E-mail: liutbejlab.org
}

\section{Haiyan Gao}

Department of Physics, Duke University, Durham, North Carolina 27708, USA

Duke Kunshan University, Kunshan, Jiangsu 215316, China

E-mail: gao@phy.duke.edu

The 3D imaging of nucleon is at the frontier of understanding the visible Universe and QCD. There are many efforts to access the nucleon's 3D partonic structure both in the transverse coordinate space (Generalized Parton Distributions) and in the transverse momentum space (Transverse Momentum Dependent distributions). The proposed Solenoidal Large Intensity Device (SoLID) in Hall A at Jefferson Lab will fully utilize the great physics potential of the 12-GeV energy upgrade by combining high luminosities and large acceptance to allow high precision measurements of the observables of interest in a wide kinematic region. We present an overview of the experiment plan and highlight some of the expected physics results.

23rd International Spin Physics Symposium - SPIN2018 -

10-14 September, 2018

Ferrara, Italy

\footnotetext{
* Speaker.

${ }^{\dagger}$ This work is supported in part by the U.S. Department of Energy, Office of Science, Office of Nuclear Physics under contract Nos. DE-FG02-03ER41231 and DE-AC05-06OR23177.
} 


\section{Nucleon 3D Structure}

The nucleon structure is one of the most active areas in Quantum Chromodynamics (QCD) which is the fundamental theory of strong interactions. While QCD can be precisely tested at high energy scales because of the small strong coupling constant due to the asymptotic freedom and the application of the perturbation theory, at low energy scales like hadrons, QCD is still unsolved due to its nonperturbative nature.

A general framework to describe the partonic structures of the nucleon is the generalized transverse momentum dependent parton distributions (GTMDs). They are related to the Wigner distribution via a transverse Fourier transform. One can obtain the generalized parton distributions (GPDs) by integrating the GTMDs over the transverse momentum. They can be viewed as the generalization of the 1D parton distribution functions (PDFs) and the 2D form factors. On the other hand, one can obtain the transverse momentum dependent parton distributions (TMDs) by setting the transferred momentum to zero or equivalently integrating the Wigner distributions over the transverse coordinate. They will further reduce to PDFs when the transverse momentum is integrated. A realistic way to directly measure GTMDs or Wigner distributions in experiments is still under theoretical explorations. However, we can access them partially by measuring the GPDs and TMDs. Both the GPDs and the TMDs are 3D parton distributions, which have a much richer spin dependence than PDFs, and can unveil some information of quark orbital angular momentum.

The $12 \mathrm{GeV}$ energy electron beam upgrade at JLab provides a unique opportunity to study the nucleon 3D structure through GPDs and TMDs, particularly in the valence quark region. It takes advantages of the complementary capabilities of different detectors in experimental Halls A, $\mathrm{B}$ and C. To fully utilize the upgrade and to maximize its discovery potentials, the Solenoidal Large Intensity Device (SoLID), capable of handling both high luminosities and large acceptance, was proposed and designed for Hall A to satisfy a highly rated and rich scientific program [1]. The SoLID GPD and TMD experiment program will be presented and we will highlight some of the expected physics results.

\section{SoLID TMD Program}

There have been many efforts to access TMDs through the semi-inclusive deep inelastic scattering (SIDIS) processes. Within the TMD factorization, SIDIS structure functions are expressed as convolutions of transverse momentum dependent parton distribution functions (TMD PDFs) and transverse momentum dependent fragmentation functions (TMD FFs). As illustrated in Figure 1, there are eight leading-twist (twist-two) quark TMD PDFs for a nucleon. If the transverse momentum is integrated, three of them, $f_{1}, g_{1 L}$, and $h_{1}$, will reduce to their collinear limits: the unpolarized PDF, helicity distribution, and transversity distribution, while the remaining five will vanish. Hence TMDs, especially the spin-dependent ones, contain much richer information than collinear PDFs, and allow us to access the correlation between quark transverse momentum and quark/nucleon spin.

SoLID SIDIS experiments involve a transversely polarized ${ }^{3} \mathrm{He}$ target, a longitudinally polarized ${ }^{3} \mathrm{He}$ target, and a transversely polarized proton target. They are designed to measure the 


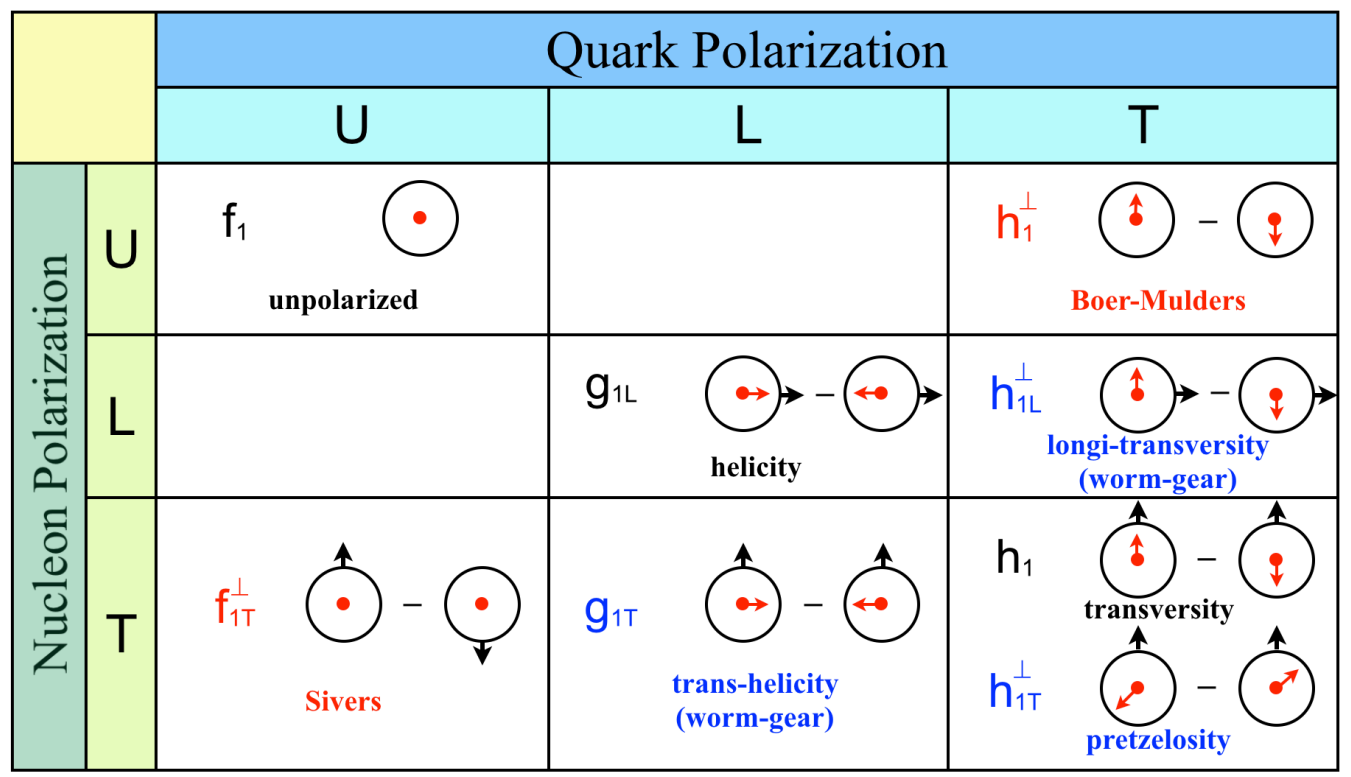

Figure 1: Eight leading-twist (twist-two) quark TMD PDFs for a nucleon.
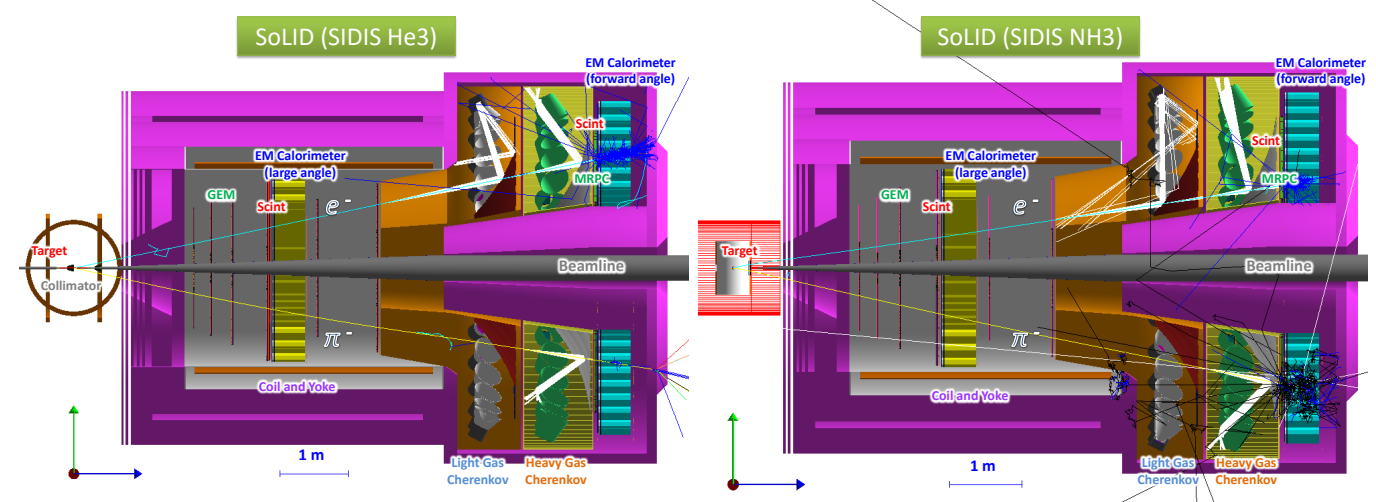

Figure 2: The experimental layout of the SoLID SIDIS setup with the ${ }^{3} \mathrm{He}$ (left) or $\mathrm{NH}_{3}$ (right) target. The scattered electrons are detected by both forward-angle and large-angle detectors. The leading pions are detected by the forward-angle detector only.

single/double spin asymmetries through the SIDIS $\left(e, e^{\prime} \pi^{ \pm}\right)$process using the SoLID spectrometer for coincident detections of the scattered electron and the produced charged pion.

The layout of the SoLID setup for SIDIS experiments is shown in Fig. 2. It consists of two sub-groups of detectors: at forward angle and at the large angle. The polar angular coverage is $8^{\circ}-15^{\circ}$ for the forward-angle detector and $15^{\circ}-24^{\circ}$ for the large-angle detector. Both of them have a full $2 \pi$ azimuthal coverage. The scattered electrons are detected by either the forwardangle or the large-angle detectors and the produced charged pions are detected by the forwardangle detector only. Two electromagnetic calorimeters made of Shashlyk type modules with good resolution and high radiation tolerance are used for electron/pion separation. A $2 \mathrm{~m}$ long light gas 
Čerenkov counter is installed at the forward angle to further improve the identification of scattered electrons. A $1 \mathrm{~m}$ long heavy gas Čerenkov counter right after the light gas Čerenkov counter can separate kaons and protons from the pions with momenta greater than $2.5 \mathrm{GeV}$. One layer of multi-gap resistive plate chamber is placed after the heavy gas Čerenkov counter to provide timing information and particle identification of hadrons at low momentum $(<2.5 \mathrm{GeV})$, as well as to suppress photon background. One layer of scintillator pad detector is placed in front of the forward angle electromagnetic calorimeter to reject photons. Another layer of scintillator pad detector is placed in front of the large angle electromagnetic calorimeter to reject photons and provide timing information and particle identification of hadrons at low momentum also. Six layers of gas electron multiplier detectors provide tracking for both sub-groups detectors in this high rate environment.

A polarized ${ }^{3} \mathrm{He}$ gas target will be used as an effective polarized neutron target with a $60 \%$ in beam polarization and a fast spin flip. Placed in front of the spectrometer entrance, it can take $15 \mu \mathrm{A}$ electron beams to reach the polarized luminosity of $10^{36} \mathrm{~N} \mathrm{~cm}^{-2} \cdot \mathrm{s}^{-1}$. To study the SIDIS process on proton, a cryogenic $\mathrm{NH}_{3}$ solid target whose protons are polarized to $70 \%$ will be placed at the same location to take $0.1 \mu$ A electron beams to reach the polarized luminosity $10^{35} \mathrm{~N} \mathrm{~cm}^{-2}$ $\mathrm{s}^{-1}$. All of SoLID sub-systems including the detectors and DAQ system are designed to handle the high luminosity with large acceptance. This is the key advantage of the SoLID SIDIS program which is capable of collecting high statistics data with well controlled systematics.

We have published the projection of SoLID SIDIS program on the $u$-quark and $d$-quark transversity and the nucleon tensor charge [2]. The expected SoLID SIDIS kinematic coverage includes: i) $0.05<x<0.6$ which comprises the majority of the valence quark region; ii) $0.3<z<0.7$ in which the leading order $x-z$ factorization is expected to hold; iii) maximum pion transverse momentum $P_{T}$ up to $1.6 \mathrm{GeV}$; and iv) $1 \mathrm{GeV}^{2}<Q^{2}<8 \mathrm{GeV}^{2}$. These kinematic coverages can be achieved by combining data with incident electron energies of 11 and $8.8 \mathrm{GeV}$. The SoLID SIDIS experiments plan to extend to a larger $x$ region with $Q^{2}$ coverage comparable with HERMES. The average statistical precision of each bin for SoLID is about $1 \%$ consisting of more than 600 bins for $p\left(e, e^{\prime}\right) \pi^{+}$ channel, compared to $37.1 \%$ (relative to the size of the asymmetry) for HERMES consisting of 7 bins in $x$ for the same channel. We have a cut of $W>2.3 \mathrm{GeV}$ to leave the feasibility of usage of low $W$ region for future developments.

The physics impact of SoLID is the precise measurement of the TMDs in the valance region. To compare the improvement on the determination of transversity, we model the transversity distributions with the recent global fit of Ref. [3], which includes the TMD evolution effect. We used simulated data for SoLID SIDIS experiments to estimate the impact of the future SoLID data on the transversity distribution of $u$ - and $d$-quarks. Our method follows Bayesian statistics where the new information is added sequentially on top of the prior knowledge without requiring a combined analysis of the old data and the new data by using the Hessian approach.

Our results for $u$ - and $d$-quark transversity distributions at $Q^{2}=2.4 \mathrm{GeV}^{2}$ are presented in Fig. 3 along with results from the global fit to existing data by Ref. [3] (KPSY15). In the upper row of plots, the uncertainties of KPSY15 are given as light shaded bands, while the projected errors after the SoLID data are taken into account are shown as dark shaded bands. To quantify the improvement of adding the future SoLID data, we show, in the bottom row of plots, the ratio of the estimated errors relative to the current errors. The results are shown using only the proton data (left panels), the neutron data (central panels), and the combination of the proton and the 

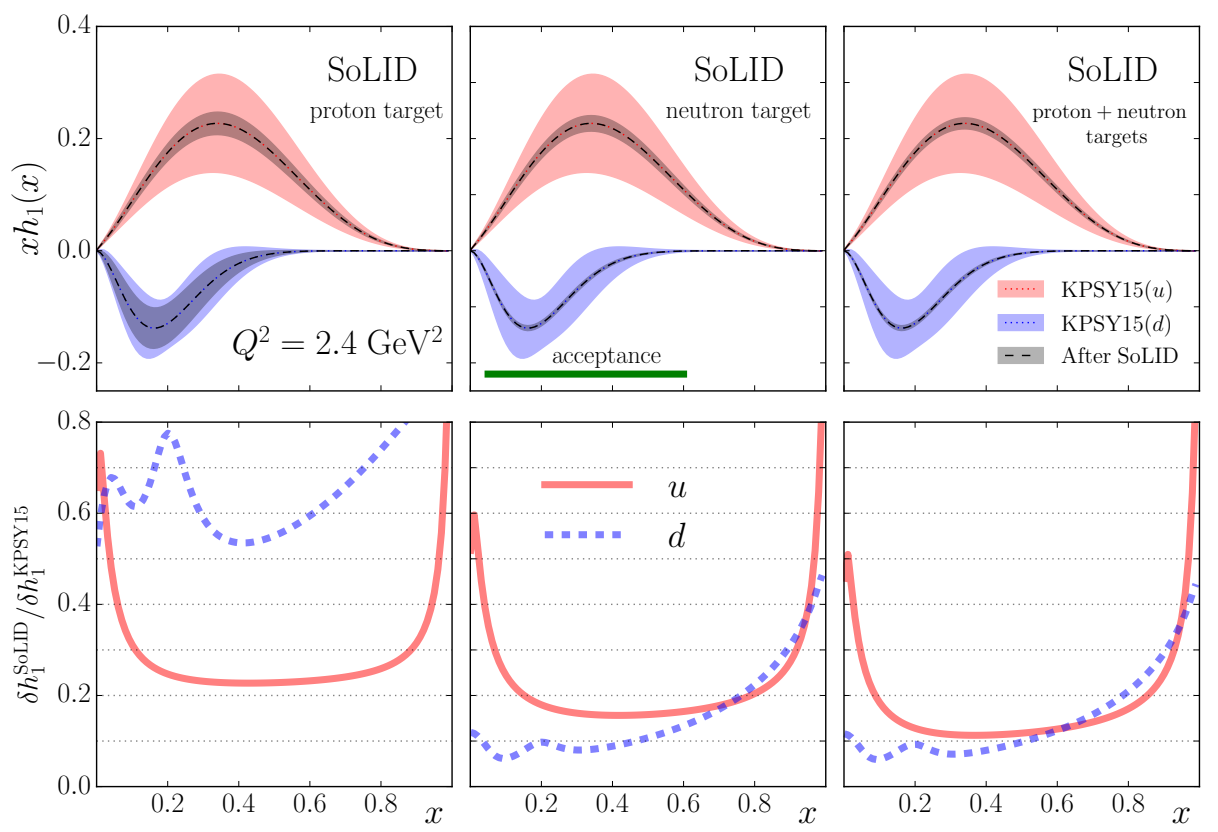

Figure 3: Upper panels: $u$-quark and $d$-quark transversity distributions at $Q^{2}=2.4\left(\mathrm{GeV}^{2}\right)$ as a function of $x$ with existing errors from KPSY15 (light shade area) and the estimated errors after the SoLID data (both statistical and systematical errors are included in quadrature) are taken into account. The acceptance region in $x$ of the SoLID experiment is indicated by the green horizontal line. Left plot: only the proton target data are taken into account, central plot: only the neutron target data are taken into account, right plot: combination of proton and neutron targets data are taken into account. Bottom panels: The ratio of the estimated errors and the current errors of transversity, $\delta h_{1}^{\mathrm{SoLID}} / \delta h_{1}^{\mathrm{KPSY} 15}$, for $u$ (solid line) and $d$ (dashed line) quarks. Left plot: the proton target, central plot: the neutron target, right plot: combination of proton and neutron targets. The "bumps" around $x \simeq 0.2$ of the $d$-quark ratio plots are artifacts of usage of Soffer positivity bound when parametrizing transversity. (The figure is from Ref. [2])

neutron data sets (right panels). While the absolute error bands can differ depending on the error analysis, the ratio of the errors is almost independent of the error analysis. Notice that the maximal improvements are attained in the region covered by the SoLID data $0.05<x<0.6$ where the errors will be reduced by approximate $90 \%$, i.e. one order of magnitude, for both $u$ - and $d$-quark transversities. The impact decreases outside of this region as expected.

The precision of the nucleon tensor charge extracted from the SoLID data will be comparable to the precision of lattice QCD calculates. It will provide a unique opportunity for searches beyond the standard model. By using the tensor charge as a bridge that relates nucleon electric dipole moments (EDMs) to quark EDMs, we can have experimental constraint on quark EDMs and thus probe additional $\mathrm{CP}$ violation sources beyond the standard model to account for the baryon number asymmetry of the universe [4].

\section{SoLID GPD Program}

GPDs are universal quantities and reflect the structure of the nucleon independently of the reaction which probes the nucleon. At leading twist-2 level, there are 4 leading-twist parton-helicity non-flip quark GPDs, $H, E, \tilde{H}$, and $\tilde{E}$. 
The GPD experiments have been mostly focused on Deeply Virtual Compton Scattering (DVCS) or Deeply Exclusive Meson Production (DEMP). DVCS depends at the same time on both the polarized $(\tilde{H}$ and $\tilde{E})$ and the unpolarized $(H$ and $E$ ) GPDs. DEMP with vector meson production is sensitive only to the unpolarized GPDs $(H$ and $E$ ), whereas pseudoscalar meson production is sensitive only to the polarized GPDs, $(\tilde{H}$ and $\tilde{E})$. In particular, information about $\tilde{E}$ via DEMP can provide new information on nucleon structure which is unlikely to be available from any other source.

SoLID DEMP experiment will detect the exclusive negative pion production from transversely polarized ${ }^{3} \mathrm{He}$ target. It will run in parallel with the SoLID SIDIS experiment. The primarily goal is the measurement of $A_{U T}^{\sin \left(\phi-\phi_{S}\right)}$. This polarization observable has been noted as being sensitive to the spin-flip generalized parton distribution $\tilde{E}$, and factorization studies have indicated that precocious scaling is likely to set in at moderate $Q^{2} \sim 2-4 G e V^{2}$, as opposed to the absolute cross section, where scaling is not expected until $Q^{2}>10 G e V^{2}$. Furthermore, this observable has been noted as being important for the reliable extraction of the charged pion form factor from pion electroproduction. The secondary goal is the measurement of the $A_{U T}^{\sin \left(\phi_{S}\right)}$ asymmetry, which is sensitive to the higher twist transversity GPDs, and provides valuable information on transverse photon contributions at small four-momentum transfer $-t$. The remaining four modulations will also be measured, but are expected to be small. The high luminosity with large acceptance of SoLID is ideal to provide a wide -t coverage for good understanding of the asymmetries.

Both DVCS and DEMP using space-like virtual probes which provides useful but yet somewhat limited view-window on GPDs. As a generalization of these programs, reactions with final state time-like photons such as Time-like Compton Scattering (TCS) or Double Deeply Virtual Compton Scattering (DDVCS), where virtual photons will decay into lepton pairs, will be powerful new tools [5].

SoLID TCS experiment will use polarized beam and a $\mathrm{LH}_{2}$ target to study the reaction $\gamma p \rightarrow$ $\gamma^{*} p^{\prime} \rightarrow e^{+} e^{-} p^{\prime}$. The differential cross section, moments of the weighted cross section, and beamspin asymmetry with circularly polarized photons will be measured as a function of the fourmomentum transfer $-t$, the outgoing photon virtuality $Q^{\prime 2}$, and the skewness $\eta$. The latter reflects the difference between the initial and final momentum fraction carried by the struck quark, and corresponds to $\xi$ in DVCS. The high luminosity of SoLID will make it possible to perform a mapping of the $Q^{\prime 2}$ - and $\eta$-dependence, which is essential for understanding factorization, higher-twist effects, and NLO corrections. It is also of particular importance for global fits aimed at extracting GPDs.

\section{Summary}

SoLID nucleon 3D imaging is a comprehensive program including SIDIS for TMDs and both DEMP and TCS for GPDs. Combining high luminosity and large acceptance, SoLID has the promise to push the TMD study to a high precision level and bring new tools for GPS study. For example, for SIDIS, the quark transversity TMD distribution can be obtained with one order of magnitude improvement over the current world data and to the precision level of lattice QCD. And for DEMP and TCS, we will be able to map asymmetries over large kinematic coverage. 
SoLID nucleon 3D imaging program will provide unprecedented precision data with a series of well planned experiments at JLab $12 \mathrm{GeV}$ era and reveal important information of nucleon structures.

\section{References}

[1] J. P. Chen et al. (SoLID Collaboration), arXiv:1409.7741 [nucl-ex] .

[2] Z. Ye et al., Phys. Lett. B 767 (2017) 91.

[3] Z.-B. Kang, A. Prokudin, P. Sun, F. Yuan, Phys. Rev. D 93 (2016) 014009.

[4] T. Liu, Z.W. Zhao and H. Gao, Phys. Rev. D 97 (2018) 074018.

[5] I.V. Anikin et al., Acta Physica Polonica B 49 (2018) 741. 